steroids therapy, the second patient underwent surgery for a malignant stricture and the third patient had enteroscopy and removal of the capsule; biopsies of the stricture were in-conclusive. The overall cohort DY for all indications was $39 \%$ $(n=377 / 958)$.

Conclusions This is the largest series from a DGH in England. Our data has shown that CE is safe, non-invasive and feasible in a district hospital setting. It has a good DY, acceptable to patient and allows adequate look at the small bowel. Recommendations: Despite the major role of CE in GI investigation, there is a lack of structured training. We recommend formal accreditation and training to be added to the Gastroenterology advance training curriculum.

\section{PTU-074 MICROSCOPIC COLITIS: INCIDENCE AND BIOPSY PATTERN IN A DISTRICT GENERAL HOSPITAL IN ENGLAND}

Hussam ME Ahmed*, Elmuhtady Said. Barnsley District General Hospital, Barnsley, UK

\subsection{6/gutjnl-2018-BSGAbstracts.453}

Introduction Microscopic colitis (MC) is characterised clinically by chronic watery diarrhoea and usually by normal-looking colonic mucosa on endoscopy. ${ }^{1}$ This creates controversy regarding the role of routine mucosal biopsy protocol. Despite an increasing incidence, understanding and awareness of $\mathrm{MC}$ remain low. The European Microscopic Colitis Group (EMCG) established a series of recommendations to enhance awareness about MC. ${ }^{2}$

Aim \& methods We aim to evaluate the incidence of $\mathrm{MC}$ in our centre; a district general hospital serving a population of 250000 in North east England. We retrospectively retrieved, reviewed and analysed data from all lower gastro-intestinal (GI) endoscopy reports and colonic biopsies histology reports for patients diagnosed with MC between January 2010 and December 2016. We assessed demographics, indications, endoscopy and biopsy histology reports of the cohort of patients with established MC.

Results 145 patients were identified. Three patients were excluded due to unavailable endoscopy reports leaving 142 eligible patients included in this service evaluation. The annual incidence rate of $\mathrm{MC}$ has increased by more than 5 folds over the six years period [Figure 1]. Females predominated the cohort with 93 patients $(65.5 \%)$ with a mean age of 61 years (range 19-85). The mean age for males was 60 years (range 19-88). 137 patients underwent colonoscopy, while only five patients had flexible sigmoidoscopy. Indications were: chronic diarrhoea $83.1 \%(\mathrm{n}=118)$, altered bowel habits $12.7 \%(n=18)$, anaemia $0.7 \%(n=1)$, per-rectal bleeding $0.7 \%$ $(\mathrm{n}=1)$, and Inflammatory Bowel Disease surveillance $2.8 \%$ $(n=4)$. Endoscopy was normal in $85.2 \%(n=121)$, while $6.3 \%$ $(n=9)$ of patients were found to have area of inflammation. Terminal ileal biopsies were performed in $12.7 \%(n=18)$ and were all negative.

Majority of patients $(55.6 \%, n=79)$ were found to have lymphocytic colitis LC, while Collagenous colitis was demonstrated in $42.3 \%(n=60)$ of patients and only $2.1 \% \quad(n=3)$ had a mixed histological picture of LC and MC reported. 89 patients $(63 \%)$ had right, left and recto-sigmoid mucosal biopsies while the rest had random mucosal biopsies.

Conclusion Data from our centre showed annual increase diagnosis of MC. TI biopsy were all negative and therefore in- conclusive in diagnosis of MC. In view of the lack of clear diagnostic biopsy protocol in normal lower GI endoscopy in patients presenting with chronic diarrhoea, either random colonic or segmental mucosal biopsies can be done to look for MC.

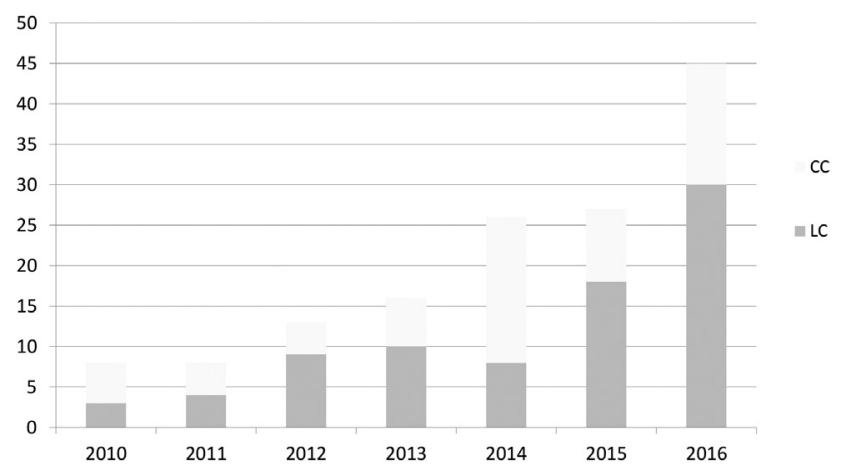

Abstract PTU-074 Figure 1 increase in the diagnosis rate of MC.

CC Collagenous colitis

LC Lymphocytic colitis

\section{REFERENCES}

1. Koulaouzidis A, Saeed AA. Distinct colonoscopy findings of microscopic colitis: Not so microscopic after all? World I Gastroenterol 2011.

2. Münch $A$, et al. EMCG. Microscopic colitis: Current status, present and future challenges: Statements of the European Microscopic Colitis Group J Crohns Colitis 2012.

\section{PTU-075 WHAT IS THE BENEFIT OF TELEPHONE AND VIRTUAL IBD CLINICS IN A DGH?}

Lulia Al-Hillawi*, Paul Blaker, Adam Harris, Bikash Gurung, Theresa Bedwell, Catriona McEnery, Eleanor Warner. Maidstone and Tunbridge Wells NHS Trust

\subsection{6/gutjnl-2018-BSGAbstracts.454}

Introduction Inflammatory Bowel Disease (IBD) services across the UK are under increasing pressure. To improve efficiency, pathways of care have been proposed. However these are difficult to cost due to a lack of data on current service provision, leading to challenges with commissioning.

We set out to characterise our IBD service in a District General Hospital (DGH) setting, having recently implemented a new pathway to streamline the service. A second aim was to establish if our service met the NICE quality standard of seeing IBD referrals within 4 weeks.

Methods Prospective data from clinics at two DGHs were gathered from 92 patient journeys over 52 weeks. The activity of a new IBD helpline was analysed over a 28 week period and outcomes prospectively recorded. Specifically the activity avoided as a result of the helpline was analysed and costed. Finally, the outcomes from a new virtual IBD clinic were prospectively collected over a 10 week period.

Results 33\% of clinic patients had IBD, of which 59\% were in remission. $41 \%$ of patients were felt suitable for non-clinic follow-up. $76 \%$ were interested in the concept of 'self-management' during remission. 95\% of patients rated the consultation experience as 'good' or 'very good'. There was an average 1 new to 4 follow-up encounters within the first year from referral; $26 \%$ were successfully conducted by telephone. Median time from initial referral to first outpatient contact was 9.1 weeks (4.9-19.9). 58\% were not seen within 4 weeks of referral. 
The IBD helpline received 543 calls in 28 weeks (average $83 /$ month). The interventions avoided due to this service were; 156 GP consultations, 231 outpatient reviews, 39 ED attendances and 6 admissions. Estimated cost saving due to activity avoided was $£ 37,913$, with helpline costs of $£ 2065$. $92 \%$ of patients rated the helpline as 'good' or 'very good'. $100 \%$ of patients reported a response within 24 hours (weekdays).

Since September 2017, 10 virtual IBD clinics have led to 191 treatment decisions. High cost biologic drugs have been stopped in 7 patients, without having to wait for face-to-face review, with an estimated saving of $£ 12300$. 59 patients were identified as needing annual review and 51 identified as needing urgent treatment decisions.

Overall quantifiable cost savings from the helpline and virtual clinic totalled $£ 11,000 /$ month.

Conclusions Telephone and virtual clinics result in earlier treatment decisions for IBD patients and give quantifiable cost savings as part of an IBD pathway at a DGH. By increasing virtual clinics and encouraging patient autonomy, we can aim to improve the NICE quality standard of seeing new IBD patients within 4 weeks of referral. Improving autonomy over services for both clinician and patient can allow for more efficient IBD care.

\section{PTU-076 USING G0OGLE SEARCH TREND DATA TO ASSESS PUBLIC INTEREST IN UPPER GI CANCER SYMPTOMS}

Lance Alleyne*, Philip Harvey, Nigel Trudgill. Sandwell And West Birmingham Hospitals, Birmingham, UK

\subsection{6/gutjnl-2018-BSGAbstracts.455}

Introduction Search engine data has been used to predict disease outbreaks based on search volumes for symptoms. Trend data can also assess information-seeking behaviour for cancer symptoms.

'Be Clear on Cancer' (BCOC) for upper GI cancers ran 26/1/ $15-28 / 2 / 15$ and resulted in increased two week wait (2ww) OGD referrals. We use Google search data to identify information-seeking behaviour for symptoms of upper GI cancers and corresponding local $2 \mathrm{ww}$ referral patterns in response to BCOC. Methods Search data from 10/2/13-3/2/18 for: 'heartburn', 'indigestion', 'reflux' and 'heartburn AND cancer' was extracted from 'Google Trends' and 'Google Adwords'. Google Trends normalised weekly search traffic to a 'relative search volume' (RSV) index between 0 ( $<1 \%$ of the peak weekly search volume) and 100 (equivalent to the highest weekly search volume during the study period).

Data was compared to monthly $2 \mathrm{ww}$ referrals for upper GI cancers at our centre over the study period. Lower GI cancer referrals were used as a control.

Results A mean annual increase in weekly RSV (compared to the annual mean RSV) for the terms 'heartburn' and 'indigestion' in the last week of December annually (from 2013 to 2017 ) of $67 \%$ and $60 \%$ respectively (figure 1). In the last week of January 2015, there was also a peak in RSV for 'heartburn' of $73 \%$, corresponding to BCOC. This peak was higher than the Christmas 2014 increase (56\%) and not replicated in the study period.

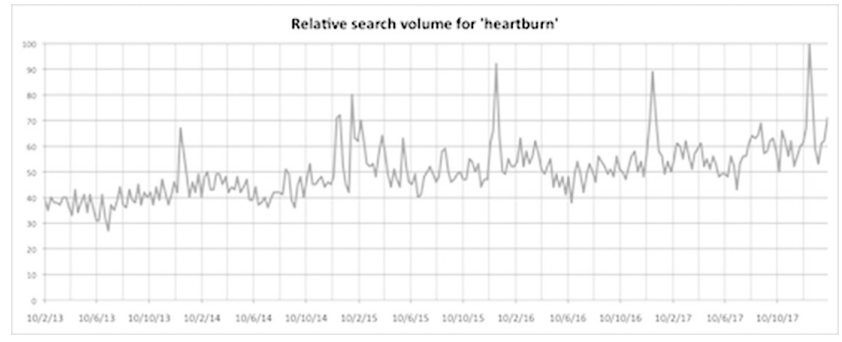

\section{Abstract PTU-076 Figure 1}

Searches for 'heartburn AND cancer' reached their peak RSV (100) in the last week of January 2015, correlating with BCOC (figure 2). Mean RSV over the entire five year reference period was 7.48 , with a $1336 \%$ increase in searches during the first week of BCOC, dropping to $722 \%$ in week $2 \%$ and $173 \%$ by week 5 - mean $673 \%$ overall.

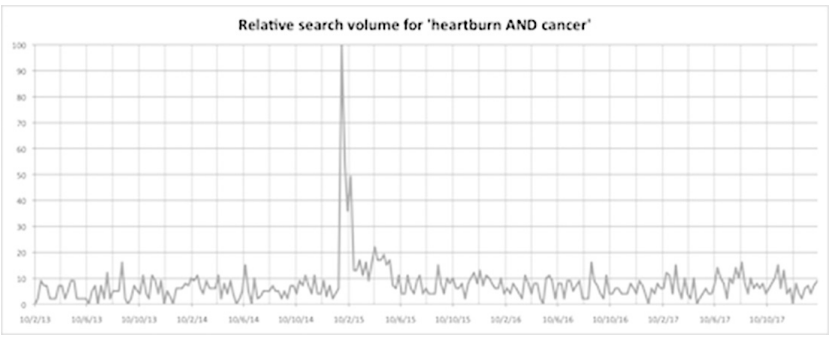

\section{Abstract PTU-076 Figure 2}

Data from our centre showed a mean 19.2\% increase in $2 \mathrm{ww}$ referrals for suspected upper GI cancers in January of every year (compared to the mean number of monthly $2 \mathrm{ww}$ referrals for the preceding 12 months), except January 2016 where there was an $11 \%$ reduction in the number of referrals. There was an $29.9 \%$ increase in $2 \mathrm{ww}$ referrals from January to February 2015 which corresponds to BCOC. Suspected colorectal cancer referrals observed a mean $3 \%$ increase each January compared to the preceding 12 month average.

Conclusions Peaks in information-seeking behaviours were observed each Christmas for upper GI symptoms resulting in a significant increase in $2 \mathrm{ww}$ referrals. BCOC generated a larger increase in interest with additional cancer association, but impact (as measured by information seeking) fell sharply over the course of the campaign.

Future BCOC programmes may benefit from 2 separate campaigns of reduced duration. Service pressures during such campaigns may be eased by such campaigns avoiding January.

\section{PTU-077 A PILOT INTERNATIONAL SURVEY OF COLONOSCOPY RELATED INJURY IN COLONOSCOPISTS}

${ }^{1}$ Ammar Al-Rifaie*, ${ }^{2}$ Stephen Hodge, ${ }^{1}$ Mo Thoufeeq, ${ }^{1}$ Mark Donnelly. ${ }^{1}$ Sheffield Teaching Hospitals, Sheffield, UK; ${ }^{2}$ Calderdale and Huddersfield Hospitals, Huddersfield, UK

\subsection{6/gutjnl-2018-BSGAbstracts.456}

Introduction Colonoscopy is physically demanding for endoscopists and for patients. Repetitive movements during colonoscopy can lead to overuse injuries. Our aim is to explore the 
prevalence and range of colonoscopy-related musculoskeletal injuries (CRI) in endoscopists.

Methods A cross-sectional electronic survey of 1825 endoscopists was made. The sample was comprised of members of British Society of Gastroenterology, European Society of Gastrointestinal Endoscopy and National Nurse Endoscopy Group. The survey comprised 20 questions. These included: endoscopists' workload, level of experience and their perceived CRIs. All endoscopists who perform colonoscopy independently were included in the analysis.

Results Initial results include 337 completed questionnaires out of $1825(18.4 \%)$. Of those, 319 (94.5\%) participants are fully independent in colonoscopy. 254 out of 319 (79.6\%) have experienced musculoskeletal injuries. These were reported as either possibly $(n=143,46.7 \%)$ or definitely $(n=90,29.4 \%)$ related to colonoscopy.

Factors that were significantly associated with higher rates of CRI: $>6$ hours per week (equivalent to 2 or more lists/week) $(p=0.0001),>5000$ life-time number of colonoscopies performed $(p=0.0002),>150$ procedure performed per year $(p=0.0001)$. Female endoscopists are also at a significantly higher risk of CRI and more likely to require time off-work $(p=0.0001)$.

Commonly injured areas were: lower back $(n=95$, $30.45 \%)$, neck $(n=90,28.85 \%)$ and left thumb $(n=80$, 25.64\%). $95(30.35 \%)$ of injured endoscopists applied some modification to their practice e.g. stretching exercises and ergonomic changes.

$130(48.5 \%)$ endoscopists thought that repetitive hand movements was a likely causative mechanism. 125 (46.6\%) believed that torqueing the scope and body position were precipitating CRI.

Several treatment modalities were used; physiotherapy $(n=109)$, medications $(n=70)$, rest $(n=43)$, splinting $(n=31)$, steroid injections $(n=26)$ and surgery $(n=11)$.

Conclusions From our initial results, a significant proportion of endoscopists experience CRI. Higher prevalence of CRI was significantly associated with $>5000$ total life-time colonoscopies, $>6$ hour/week performing colonoscopy, $>150$ procedure/ year and female gender. These results highlight the need to recognise CRI as an important issue and to adopt preventative strategies routinely in the future.

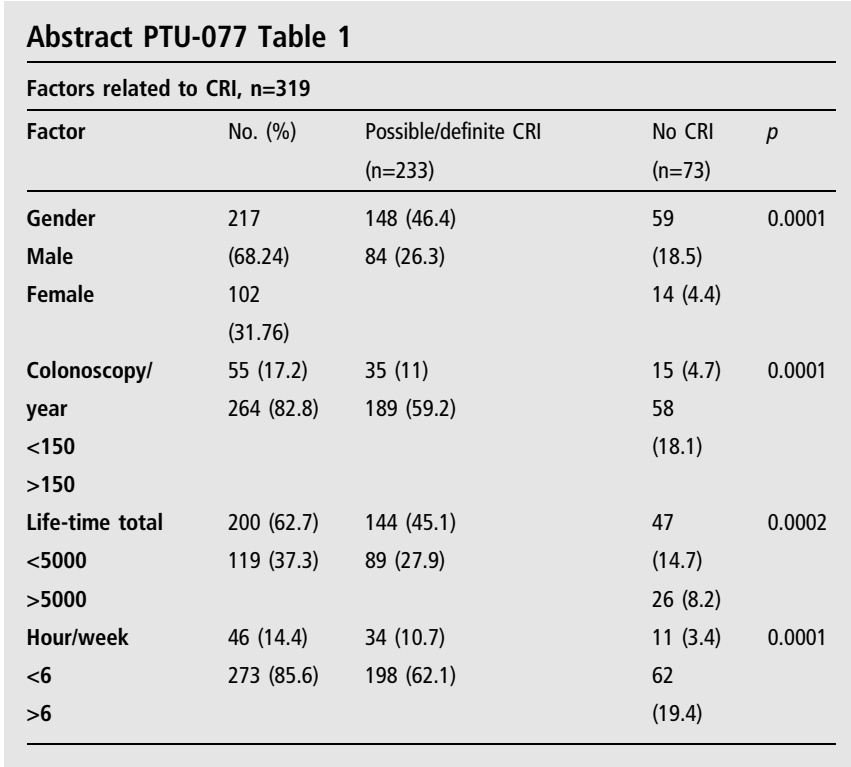

\section{PTU-078 COMPREHENSIVE MAPPING OF QUALITY STANDARDS} (QSS) IN GASTROENTEROLOGY (GI)

Rebecca Anderson*, John Anderson. Gloucestershire Hospitals NHS Foundation Trust, Cheltenham, UK

\subsection{6/gutjnl-2018-BSGAbstracts.457}

Introduction Audit is a vital part of quality assurance and enables individuals and services to monitor and improve standards. We describe the experience of mapping and implementing a comprehensive GI audit programme.

Methods Different GI QSs are published by the British Society of Gastroenterology, Joint Advisory Group on GI endoscopy (JAG), National Institute for Clinical Excellence and National Confidential Enquiry into Patient Outcome and Death. These were reviewed, where necessary rationalised (overlap/repetition), and grouped by subspecialty. An audit lead was appointed for each subspecialty.

Existing QS audit activity was mapped and compared to the new QS template. Audit-leads then designed a comprehensive GI audit programme incorporating all QSs, which was integrated into a rolling calendar of audit activity.

Results We identified 226 QSs related to GI. 101 were removed (clinical, surgical or community domains), leaving 125 GI relevant QSs (table1).

\begin{tabular}{ll} 
Abstract PTU-078 Table 1 & Subspecialty QSs \\
\hline Subspecialty & No QSs \\
\hline Bowel cancer screening & 6 \\
Endoscopic retrograde cholangiopancreatography and endoscopic ultrasound & 16 \\
Hepatology and alcohol use & 18 \\
Inflammatory bowel disease & 12 \\
JAG & 42 \\
Nutrition, percutaneous endoscopic gastrostomy and coeliac & 12 \\
Other endoscopy (Barrett's/dyspepsia) & 4 \\
Upper gastrointestinal bleeding & 15 \\
Total & 125 \\
\hline
\end{tabular}

97/125 (77.6\%) QSs were being actively audited (within 12 months), increasing to $109 / 125$ (87.2\%) over the past 5 years.

Currently $55 / 125$ can be audited electronically, 24/125 require notes review and 46/125 involve a combined approach. Compliance was poor for QSs needing notes review (49/70), but increased significantly with electronic audit data $(51 / 55 ; \mathrm{p}=0.001)$ (figure 1$)$.

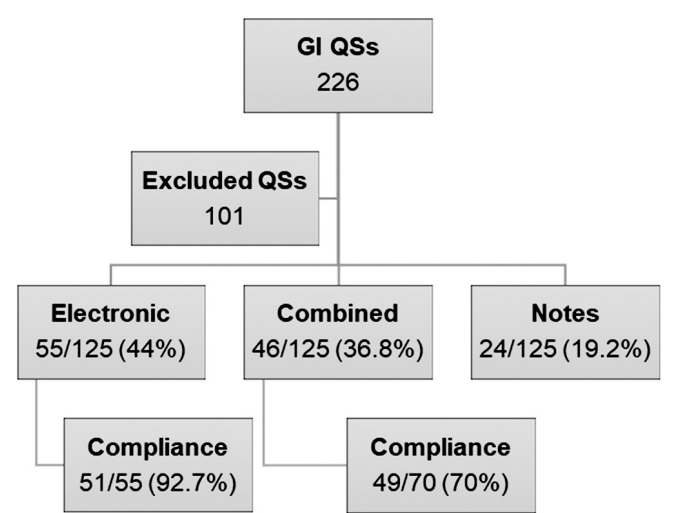

Abstract PTU-078 Figure 1 Existing QS audit compliance 